\title{
Biodegradación Temprana en Suelo de Biocompuestos Elaborados con Poli-3-hidroxbutirato, Policaprolactona y Aserrín de Cannabis Indica-Rusia Blanca
}

\author{
Oscar Y. Buitrago(1)*, Pedro J. Rodríguez ${ }^{(1)}$ y Mario J. Monroy ${ }^{(1)}$ \\ (1) Facultad de Ingeniería Universidad Militar Nueva Granada, carrera 11 No. 101-80. Bogotá-Colombia. \\ (e-mail: oscar.buitrago@unimilitar.edu.co)
}

${ }^{*}$ Autor a quien debe dirigirse la correspondencia

Recibido Mar. 14, 2018; Aceptado May. 25, 2018; Versión final Jun. 15, 2018, Publicado Dic. 2018

\begin{abstract}
Resumen
Se prepararon dos biocompuestos de poli-3-hidroxibutirato/policaprolactona/aserrín de cannabis sativa indicarusia blanca con relación en peso 70/30/(0y30) respectivamente. Los compuestos fueron mezclados en molino de rodillos, se obtuvieron láminas mediante moldeo por compresión. Probetas de cada material se enterraron en suelo durante cuatro meses. Finalizado el periodo, las probetas fueron desenterradas y se sometieron a análisis de espectroscopia de infrarrojo, difracción de rayos $\mathrm{X}$, microscopía de barrido electrónico, ensayos de tensión y pérdida de masa. Los biocompuestos que fueron enterrados en el suelo presentaron cambios en la estructura química y cristalina, disminución de propiedades mecánicas, pérdida de masa y deterioro superficial con respecto a las muestras testigo. Los resultados aportan información relevante acerca de la biodegradación inicial de compuestos fabricados con biopolímeros y aserrín obtenido del tallo de marihuana cultivada legalmente para aplicación médica. En esta investigación se genera conocimiento enfocado a la elaboración de compuestos $100 \%$ biodegradables.
\end{abstract}

\section{Early Biodegradation in Soil of Biocomposites Made with Poly- 3-hydroxybutyrate, Polycaprolactone and Sawdust of Indica- White Russian Cannabis}

\begin{abstract}
Biocomposites based on poly-3-hydroxybutyrate/polycaprolactone/cannabis sativa indica-Russia white sawdust $70 / 30 /(0$ and 30$)$ parts by weight were prepared. Mixing was carried out on a conventional laboratory two roll mill. Sheets of biocomposites were manufactured by compression molding. Specimens of each compound were buried in soil for four months. At the end of the period, the specimens were taken out of the soil and subjected to infrared spectroscopy, X-ray diffraction, electron scanning microscopy, tensile tests and mass loss. The biocomposites that were buried in the soil presented changes in the chemical and crystalline structure, decrease in mechanical properties, loss of mass and surface deterioration with respect to the control samples. The results of this study provide relevant information about the initial biodegradation of biocomposites manufactured with sawdust obtained from the stem of legal marijuana crops (for medical application). This paper is intended to promote the manufacture of $100 \%$ biodegradable polymeric compounds.
\end{abstract}




\section{INTRODUCCIÓN}

Actualmente los polímeros biodegradables se producen para reemplazar poliolefinas, disminuir la demanda de petróleo y atenuar la acumulación en el planeta. Son producidos por microorganismos que utilizan fuentes de carbono como azúcar, maíz, soya, aceites vegetales, glicerol y desechos orgánicos. Destacan el poliácido láctico, polihidroxialcanoatos (PHAs) y un grupo de sintetizados a partir del petróleo, como el adipatotereftalato de polibutileno, polibutileno succinato y policaprolactona (PCL). Hay continuo desarrollo enfocado en el mejoramiento de propiedades mecánicas y de procesabilidad de bio-plásticos (Coutinho de Paula et al., 2017; Meeks et al., 2015; Mei et al., 2017; Mohanty et al., 2000; Nwinyi y Owolabi, 2017; Scaffaro et al., 2009; Vanegas y Ramírez, 2016). En esta investigación se fabricaron compuestos a partir de poli-3-hidroxibutirato $\mathrm{P}(3 \mathrm{HB})$ con $\mathrm{PCL}$ y aserrin de cannabis, midiendo propiedades mecánicas, caracterizacion morfológica y estructura química relacionadas con la biodegradación en suelo por un periodo corto.

EI $\mathrm{P}(3 \mathrm{HB})$ es un polihidroxialcanoato corto, altamente cristalino, de resistencia similar al propileno, duro, frágil y difícil de procesar porque sus temperaturas de ablandamiento y degradación son cercanas. Este biopolímero es de fácil biodegradación, resistente la humedad, además ofrece excelente barrera a gases y radiación uv (Ali et al., 2018; Montano-Herrera et al., 2014; Urtuvia et al., 2014). Se sintetiza por los microorganismos Bacillus spp, alcaligenes latus, alcaligenes eutrophus, ralstonia eutropha, azotobacter vinelandii $y$ azotobacterchroococum (Kovalcik et al., 2017; Loureiro et al., 2014) y para mejorar procesabilidad se mezcla con poliglicidil metacrilato, polivinil pirrolidona, polibutilen succinato, polibutadieno co-acrilonitrilo, almidón, chitosan, acetato butirato de celulosa entre otros (Ke et al., 2017; Sun et al., 2017). Es biodegradado por bacterias y hongos produciendo aeróbicamente $\mathrm{CO}_{2}$ y agua y anaeróbicamente $\mathrm{CH}_{4}$ y agua (Yean y Sudesh, 2016). A la intemperie se oxida, foto-degrada e hidroliza (Mohanty et al., 2000; López et al., 2011; Urtuvia et al., 2014; Volova et al., 2010).

A diferencia del $\mathrm{P}(3 \mathrm{HB}), \mathrm{PCL}$ se sintetiza a partir de reacciones de apertura de anillo y polimerización de $\varepsilon^{-}$ caprolactona con catalizadores como ocatanoato estañoso e iniciadores que contienen átomos activos de hidrógeno (Mohanty et al., 2000). Es un polímero alifático, semicristalino, semirrígido a temperatura ambiente que se degrada totalmente entre 2-4 años según su peso molecular. Posee buena fuerza mecánica y remarcable tenacidad, además es flexible e hidrófoba (Boyandin et al., 2013; Sun et al., 2017). Debido a su alta ductilidad y fluidez se puede mezclar con otros biopolímeros para mejorar procesabilidad. Se han probado con éxito mezclas entre PHAs y PCL en aplicaciones de ingeniería biomédica, (Abdelrazek et al., 2016; Ali Akbari Ghavimi et al., 2015; Correa et al., 2017; Valdés et al., 2016; Wachirahuttapong et al., 2016).

En cuanto a mezclas de biopolímeros con fibras naturales, estas son un verdadero biocompuesto pues tanto la fase continua como la dispersa son biodegradables. Las fibras vegetales ofrecen características como producción sostenible, menor densidad respecto a cargas minerales y baja abrasión sobre la maquinaria de proceso (Liu et al., 2017; Mohanty et al., 2000; Valdés et al., 2016).

El principal inconveniente al usar fibras o harinas vegetales consiste en la baja adhesión interfacial con los polímeros (por su naturaleza hidrófila debida a los grupos $(-\mathrm{OH})$ de la celulosa y lignina), que se traduce en disminución de resistencia a la tracción, al impacto y deformación a rotura, Como solución se han propuesto tratamientos superficiales que incluyen inmersión en acido acético, $\mathrm{NaOH}$ y $\mathrm{H}_{2} \mathrm{O}_{2}$, entre otros (Kwon et al., 2014). Sin embargo los mejores resultados se han obtenido usando agentes de acoplamiento tipo copolímeros con ramificaciones funcionales (maleatos, acrilatos) que crean enlaces entre la celulosa y la cadena del polímero, aumentando la compatibilidad (Liu et al. 2017; Mohanty et al., 2000).

Respecto a la fibra vegetal empleada en este trabajo y que se denomina AcANAB, consiste en aserrín obtenido a partir de los tallos de una variedad híbrida de Cannabis indica-rusia blanca, los cuales se consideran subproducto de cultivos de Cannabis reglamentados por el Gobierno Colombiano para fines medicinales y de investigación. En general, las plantas de Cannabis indica poseen tallos leñosos, diferenciándose del cáñamo (Cannabis sativa L) el cual produce fibras largas de alto desempeño mecánico (Cirovic et al., 2017; Liu et al., 2017; Rahman et al., 2011; Singha y Rana 2012; Srinivasababu, 2014).

En este estudio, se elaboraron dos tipos de biocompuestos a partir de $\mathrm{P}(3 \mathrm{HB})$ y $\mathrm{PCL}$ en relación 70:30 en peso respectivamente, el primer compuesto se elaboró sin $A_{C A N B}$, a la segunda mezcla se le agregaron 30 partes por cien de polímero de A durante cuatro meses, después de este periodo fueron analizadas a través de microscopia de barrido electrónico, difracción de rayos $\mathrm{X}$, propiedades de tensión y espectroscopia de infrarrojo con la finalidad de medir el avance de la biodegradación. Los resultados fueron comparados contra probetas testigo. Tambien se analizó el efecto de la incorporación de aserrín sobre propiedades de tensión. 


\section{METODOLOGÍA}

Para el desarrollo del proyecto, se utilizó como fibra natural aserrín (harina) de tallos de cannabis indica-rusia blanca, buscando alternativas de aprovechamiento pues los mismos son considerados desecho. A los biocompuestos obtenidos se les realizaron diferentes pruebas de caracterización antes y después de enterrarlos en suelo.

\section{Materiales}

$\mathrm{P}(3 \mathrm{HB})$ referencia PHA $211080^{\circledR}$, densidad $1,25 \mathrm{~g} / \mathrm{cm}^{3}$, TABLANDAmIENTO $170 \stackrel{\circ}{\circ} \mathrm{C}$, índice de fluidez: $3,4 \mathrm{~g}(10 \mathrm{~min}, 170$ ${ }^{\circ}$ C) comprado a Hangzhou Ruijiang Chemical Co. Ltd. PCL con peso molecular 70.000 , densidad $1,15 \mathrm{~g} / \mathrm{cm}^{3}$, índice de fluidez: $1900 \mathrm{~g}\left(10 \mathrm{~min}, 80^{\circ} \mathrm{C}\right)$; TABLANDAMIENTO $60 \stackrel{\circ}{\circ} \mathrm{C}$ comprada a Taian Health Chemical Co. Ltd. El aserrín fue obtenido del tallo de cannabis indica-rusia blanca cultivada en Corinto-Cauca-Colombia, el análisis bromatológico fue realizado por el laboratorio Agrilab $^{\circledR}$ Bogotá D.C.-Colombia (Figura 1a) y la distribución de tamaño de partícula se hizo por difracción laser en el equipo Mastersizer 3000 Malvern Instruments ${ }^{\circledR}$ (Fi.1b).
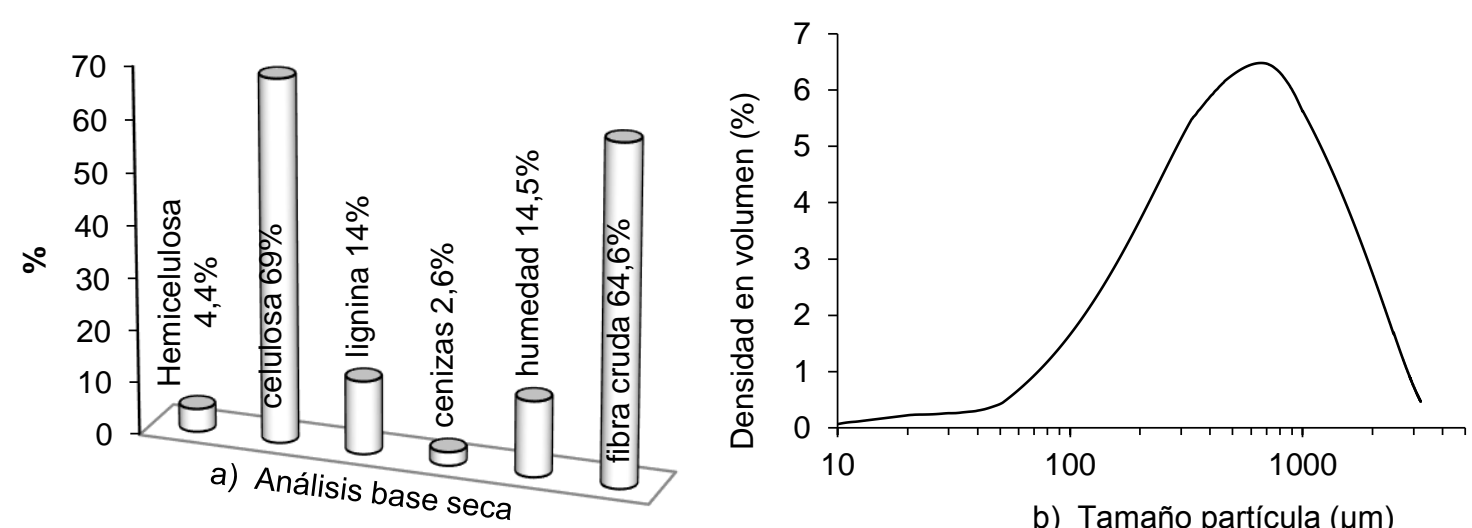

Fig. 1: Caracterización AcANAB. a) Bromatología. b) Distribución tamaño de partícula

\section{Elaboración de biocompuestos}

Los dos biomateriales hechos se denominaron P0 y P30. P0 se formuló con 70 pcp de $\mathrm{P}(3 \mathrm{HB})$ más 30 pcp de PCL, sin $A_{C A N A B}$; y P30: contiene 70 pcp de $\mathrm{P}(3 \mathrm{HB})$ más $30 \mathrm{pcp}$ de $\mathrm{PCL}$ más $30 \mathrm{pcp}$ de Acanab (ver Tabla 1). En la Figura 2 se muestra el aserrín $A_{C A N A B}$, el compuesto mezclado y la lámina de biocompuesto $P 30$. Ambas mezclas fueron elaboradas en un mezclador de rodillos con sistema de calentamiento; $\mathrm{kg} / \mathrm{batch}: 1,0$ gramos. Especificaciones de rodillos: diámetro $12 \mathrm{~cm}$, longitud $35 \mathrm{~cm}$, relación de fricción 1,4:1.

Tabla 1: Formulación de mezclas

\begin{tabular}{|l|c|c|}
\hline \multirow{2}{*}{ Ingredientes } & \multicolumn{2}{|c|}{ Partes por cien de polímero (pcp) } \\
\cline { 2 - 3 } & $P 0$ & $P 30$ \\
\hline $\mathrm{P}(3 \mathrm{HB})$ & 70 & 70 \\
\hline $\mathrm{PCL}$ & 30 & 30 \\
\hline Aserrín de cannabis-indica-rusia blanca & 0 & 30 \\
\hline Acido esteárico (ayuda de proceso) & 1 & 1 \\
\hline
\end{tabular}

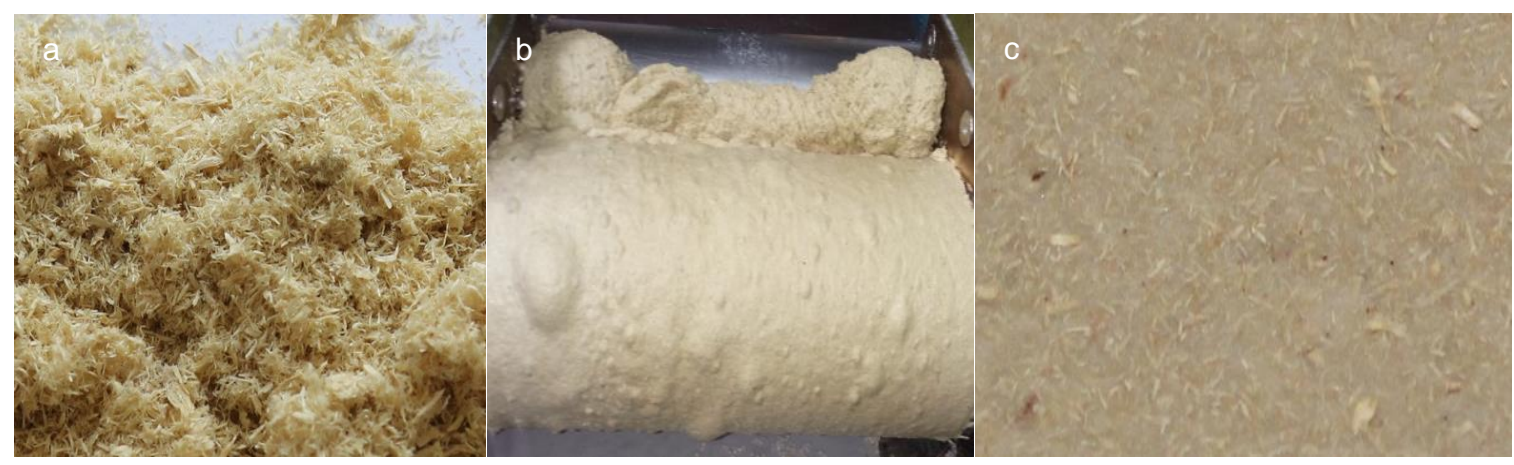

Fig. 2: P30. a) ACANB, b) Mezclado del compuesto en molino de rodillos, c) Biocompuesto P30 en lámina 
El mezclado se inició con los rodillos precalentados a $130 \pm 2 \stackrel{\circ}{ }$, se agregó PCL para formar una banda, se adicionó lentamente $\mathrm{P}(3 \mathrm{HB})$ hasta derretimiento completo (5 min). Para el compuesto P30 se incorporó lentamente $A_{C A N A B}(5 \mathrm{~min})$, se adicionó la ayuda de proceso, se homogenizó la mezcla y se descargó (3 min), tiempo global 13 minutos. Las láminas de biocompuesto para elaborar probetas se obtuvieron mediante moldeo por compresión en una prensa hidráulica con calentamiento eléctrico en los platos. Parámetros de proceso: área molde $16^{*} 16 \mathrm{~cm}^{2}$; espesor molde $3,3 \mathrm{~mm}$, cargue $110 \mathrm{~g}$; temperatura del molde: $150 \pm 1 \stackrel{\circ}{\circ}$; presión: 200 kgf/cm²; diámetro del pistón: $10 \mathrm{~cm}$; tiempo de prensado: 4 minutos.

\section{Ensayo de biodegradación en suelo}

Mezclas P0 y P30 en forma de probetas tipo I (ASTM D638-10) se enterraron a profundidad de $30 \mathrm{~cm}$ en un campo cubierto de pasto, con coordenadas de latitud norte $4^{\circ} 45^{\prime} 49,032^{\prime \prime} ; 58^{\circ}$ longitud oeste $73^{\circ} 36^{\prime} 36,18^{\prime \prime}$; en zona de montaña a 2400 m.s.n.m., temperatura promedio de $16{ }^{\circ} \mathrm{C}$, a $57 \mathrm{~km}$ sentido noreste de Bogotá D.C. (Colombia). La biodegradación fue llevada a cabo durante cuatro meses, desde octubre 02 de 2017 hasta febrero 02 de 2018, con periodos alternos de lluvia y clima seco. Las probetas desenterradas se denominaron P0-D y P30-D respectivamente (Figura 5), fueron limpiadas con cepillo de cerdas metálicas, se lavaron vigorosamente con agua destilada y fueron secadas a $45^{\circ} \mathrm{C}$ en un horno a vacío durante 24 horas.

\section{Ensayos de Tensión}

Para medir el avance de biodegradación además de la incorporación de 30 pcp de ACANB, se fabricaron probetas con geometría tipo I (norma ASTM D638-10), se usó una maquina universal de tensión Shimadzu ${ }^{\circledR}$ AGS-X con celda de carga de $2,0 \mathrm{kN}$, la velocidad de estiramiento en los ensayos se mantuvo constante a 50 $\mathrm{mm} / \mathrm{min}$. Previamente las probetas fueron acondicionadas a $22{ }^{\circ} \mathrm{C}$ y $51 \%$ de humedad relativa durante 24 horas. Se analizaron la resistencia a la tracción, deformación a rotura y módulo de Young.

\section{Microscopia de barrido electrónico (SEM)}

Cambios en la morfología de los compuestos antes y después de biodegradación fueron analizados a través de imágenes SEM, las cuales fueron tomadas a una magnificación de $500 \%$ en un microscopio de barrido electrónico JEOL JCM-5000 NeoScope ${ }^{\mathrm{TM}}$. Previamente las muestras fueron cubiertas con película de grafito para incrementar la conductividad térmica y garantizar una correcta toma de imágenes.

\section{Espectroscopia infrarroja (FT-IR)}

Con el fin de verificar cambios en la estructura química debida a la biodegradación, se analizaron espectros infrarrojos de los materiales P0; P0-D; P30, P30-D en un espectrómetro Nicolet iS10 Thermo Fisher Scientific, en el intervalo de longitud de onda de $500 \mathrm{~cm}^{-1}$ hasta $4000 \mathrm{~cm}^{-1}$. 32 scan fueron tomados por espectro con resolución de $4 \mathrm{~cm}^{-1}$. Las pastillas se prepararon mezclando $80 \mathrm{mg}$ de $\mathrm{KBr}$ con 4,0 mg de biocompuesto.

\section{Difracción por rayos $X(X D R)$}

La desaparición de fase cristalina en biocompuestos por biodegradación se puede evaluar con difractometría de rayos X (Volova et al., 2010). Se hicieron mediciones en ARL ${ }^{T M} 9900$ X-Ray WorkStation ${ }^{T M}$ Thermo Fisher Scientific. Los datos difractométricos se procesaron con el software Oxsas ${ }^{\circledR}$, con longitud de onda 1,54 $\AA$; temperatura $21^{\circ} \mathrm{C}$, voltaje $40 \mathrm{kV}$; barrido del ángulo $2 \theta: 0,03$ desde $8^{\circ}$ hasta $80^{\circ}$.

Pérdida de masa

Otra manera de evaluar la biodegradación es cuantificar la pérdida de masa (Scaffaro et al., 2009). La variación fue calculada como porcentaje de variación entre el peso inicial y final, según la ecuación 1.

$$
M_{\%}=\frac{M_{I}-M_{F}}{M_{I}} * 100
$$

Dónde: $M_{\%}=$ Pérdida porcentual de masa; $M_{l}=$ peso inicial del compuesto $M_{F}=$ peso del compuesto después de cuatro meses.

\section{RESULTADOS Y DISCUSIÓN}

PCL se mezcló con $\mathrm{P}(3 \mathrm{HB})$ buscando mejorar procesabilidad, disminuir fragilidad e imprimir ductilidad a las mezclas P0 y P30. Una ventaja de la adición de AcanB es su baja densidad $\left(0,84 \mathrm{~g} / \mathrm{cm}^{3}\right)$ frente a $1,22 \mathrm{~g} / \mathrm{cm}^{3}$ (densidad P0). Al incorporar 30 pcp de AcANB (P30) se obtuvo un compuesto 10\% más liviano respecto a P0. Ensayos de tensión 
La evaluación de la biodegradación y el efecto de la adición de AcANAB sobre propiedades de tensión se realizó partiendo de un diseño experimental completamente al azar de un factor con cuatro niveles (P0, P30, P0-D y P30-D), la variable de salida corresponde a: resistencia a la tracción, deformación a rotura y módulo de Young, empleando cuatro (4) observaciones por cada ensayo. Los resultados fueron juzgados aplicando ANOVA de una vía, se usó Statgraphics ${ }^{\circledR} 5.1$ Plus con 95\% de confianza y las diferencias entre las medias fueron analizadas aplicando HSD Tukey (ver Tabla 2). El análisis estadístico permite afirmar que hubo diferencias significativas $(p<0,05)$ entre las propiedades de tensión de cada compuesto antes y después de ser enterrados en el suelo por 4 meses. También se observan en la Tabla 2, diferencias significativas entre los compuestos que no fueron sometidos a biodegradación (P0 y P30), debidas a la incorporación de AcANB.

Tabla 2: ANOVA de una vía. Ensayos de Tensión

\begin{tabular}{|l|c|c|c|c|c|l|c|c|}
\hline Respuesta & $p$ & $\begin{array}{c}\text { Contraste de } \\
\text { medias HSD } \\
\text { Tukey }\end{array}$ & Respuesta & $p$ & $\begin{array}{c}\text { Contraste de } \\
\text { medias HSD } \\
\text { Tukey }\end{array}$ & Respuesta & $\begin{array}{c}\text { Contraste de } \\
\text { medias HSD } \\
\text { Tukey }\end{array}$ \\
\hline Tracción & 0.000 & $\begin{array}{l}\text { P0 y P0-D; P0 y } \\
\text { P30; P0 y P30-D; } \\
\text { P30 y P30-D }\end{array}$ & $\begin{array}{c}\text { Deformación } \\
\text { a rotura }\end{array}$ & 0,0001 & $\begin{array}{c}\text { P0 y P0-D; P0 } \\
\text { y P30; P0 y } \\
\text { P30-D }\end{array}$ & $\begin{array}{l}\text { Módulo } \\
\text { Young }\end{array}$ & $\begin{array}{l}\text { 0.0000 P0 y P0-D; P0 y } \\
\text { P30; P0 y P30- } \\
\text { D; P30 y P30-D }\end{array}$ \\
\hline
\end{tabular}

\section{Aserrín de cannabis, P0 contra P30}

La adición de 30 pcp AcAnB disminuyó la resistencia a la tracción en 49,3\% y la deformación a rotura en 95,8\%, con respecto a P0 (ver Tabla 3). Estos resultados guardan relación con los reportados por (Valdés et al., 2016), quienes reportan disminución del $97 \%$ de la propiedad de elongación al incorporar $30 \%$ de cascarilla de almendras con PCL.

Tabla 3: Propiedades físico-mecánicas, tabla de medias

\begin{tabular}{|l|c|c|c|}
\hline Muestra & Tracción (MPa) & Deformación a rotura (\%) & Módulo de Young (MPa) \\
\hline P0 & $14,49 \pm 0,46$ & 498,350 & $38,33 \pm 2,52$ \\
\hline P0-D & $4,00 \pm 0,465$ & 29,198 & $24,12 \pm 2,52$ \\
\hline P30 & $7,35 \pm 0,46$ & 21,050 & $69,25 \pm 2,52$ \\
\hline P30-D & $4,27 \pm 0,46$ & 9,345 & $50,99 \pm 2,52$ \\
\hline
\end{tabular}

La pérdida de la deformación a rotura (ver Figura 3) puede considerarse independiente de la distribución de tamaño de partícula. Para el aserrín de cannabis el intervalo de diámetro fue ancho (50-2000 $\mu \mathrm{m})$ mientras que (Valdés et al., 2016) reportaron distribución estrecha de tamaño de partícula para la cascarilla de almendras $(40-180 \mu \mathrm{m})$.

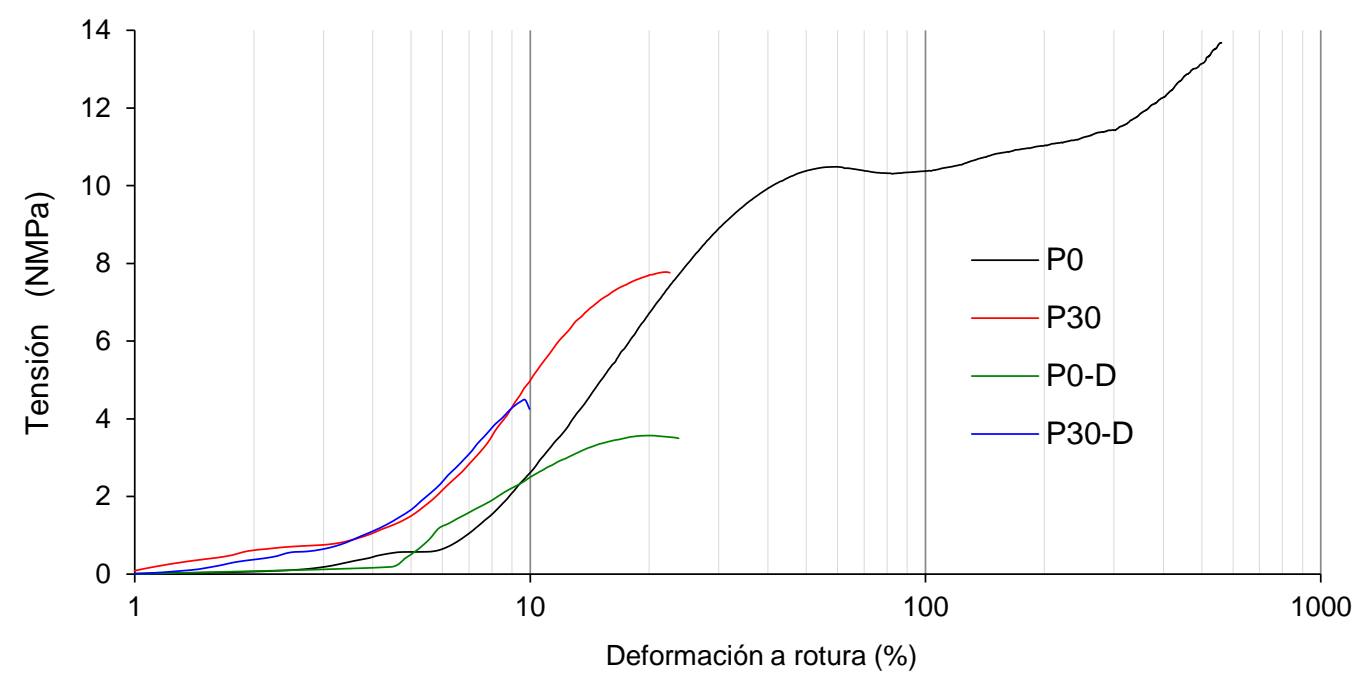

Fig. 3: Curva típica tensión-deformación. Efecto de biodegradación temprana en suelo de compuestos P0 y P30

La disminución de resistencia a la tracción y deformación debido a incorporación de AcANB es atribuible a baja compatibilidad entre la partícula (hidrófila) y polímero (generalmente hidrófobo), la adhesión en la interface polímero-partícula fue débil, por tanto, los esfuerzos no se propagaron en esta zona, al contrario, en la unión 
se produjo rotura o quiebre. Para evitar que la elongación disminuya drásticamente se adoptan estrategias para impartir adhesión interfacial aplicando agentes de acoplamiento (Cocca et al., 2015). El módulo de elasticidad aumentó $80 \%$ al agregar 30 pcp de ACANB con relación a la mezcla P0 (ver Tabla 2), las fibras naturales por lo general tienen alto módulo de elasticidad e imparten rigidez cuando se mezclan con polímeros (Palacio et al., 2016).

\section{Cambio en las propiedades de tensión debido a biodegradación}

Las probetas de compuestos sometidos a biodegradación en suelo (P0-D y P30-D) presentaron disminución significativa en las propiedades tracción, elongación y módulo de elasticidad (ver Tabla 1 y Figura 3), siendo P0-D la muestra que arrojó los más altos valores de perdida de propiedades: tracción 94,1\%; elongación $65,2 \%$; módulo de Young 37,1\%. Se evidenció perdida de ductilidad en las probetas enterradas.

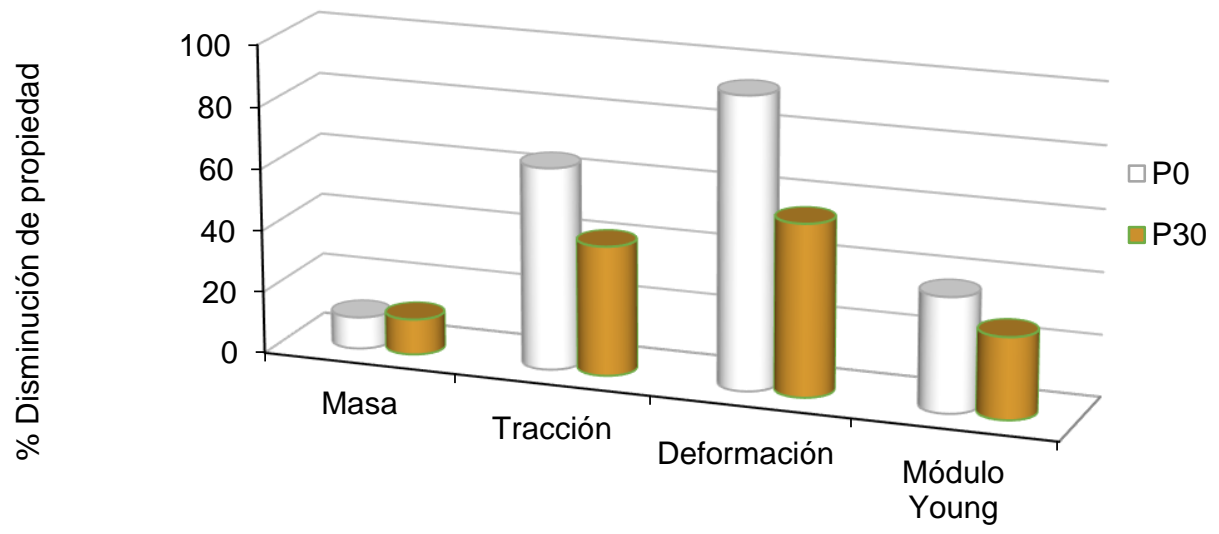

Fig. 4: Disminución de propiedades de biocompuestos P0, P30 sometidos a biodegradación temprana en suelo

Pérdida de masa

El biocompuesto P0 perdió 10,2\% de masa y P30 10,2\% (ver Figura 4), la pérdida de peso fue similar lo que indica que después de 4 meses hay degradación lenta pero progresiva. Hay reportes de biodegradación acelerada por vía enzimática con pérdida de masa de hasta $70 \%$ en 25 días para compuestos de PCL con residuos industriales (Valdés et al., 2016), y con lodos activados en 4 meses un biocompuesto de PCL/almidón (Mater-biं ${ }^{\circledR}$ ) con harina de madera presentó $70 \%$ de pérdida de peso (Scaffaro et al., 2009).

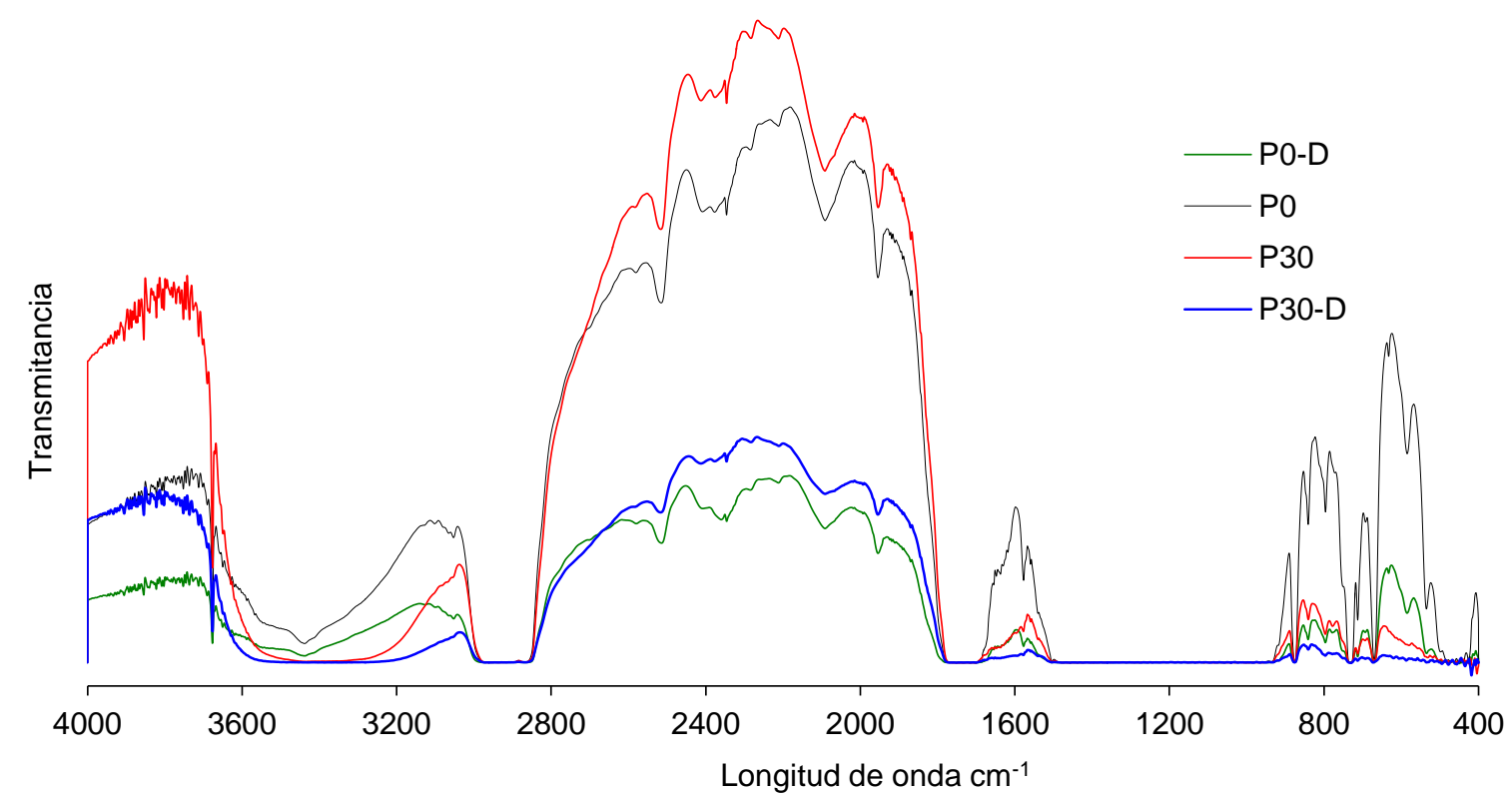

Fig. 5: FT-IR. Espectros comparativos de compuestos antes y después de biodegradación en suelo (4meses)

\section{Análisis de biodegradación por espectroscopia infrarroja FT-IR}

Los resultados de disminución de propiedades mecánicas y pérdida de peso indican que hay inicio de biodegradación de las mezclas P0, P30, con cambios en la estructura química del compuesto como 
corroboran los espectros de infrarrojo (ver Figura 5). La vibración axial del OH observada en $3300-3500 \mathrm{~cm}^{-1}$ corresponde al grupo saliente del $\mathrm{P}(3 \mathrm{HB})$, al hidroxilo de celulosa y lignina. Por lo tanto, $\mathrm{OH}$ es característico en los espectros P0 y P30 y se observó disminución de la intensidad de esta banda en ambos compuestos sometidos a biodegradación temprana.

Esto evidencia cualitativamente la rotura del enlace $\mathrm{OH}$ del biopolímero según se observa en el espectro de P0-D. Desde luego que también en P30 se observó disminución en la intensidad de la banda, sin embargo en este compuesto no fue posible identificar si la pérdida del enlace $\mathrm{OH}$ ocurrió tanto en el polímero como en la celulosa y lignina. Otro enlace característico es $\mathrm{C}=\mathrm{O}$, presente en ambos biopolímeros. La vibración de estiramiento se ubicó en 1630-1750 $\mathrm{cm}^{-1}$, y es evidente una disminución considerable de este grupo en el espectro P0-D. El comienzo de la biodegradación es atribuible a una escisión del enlace ester por la acción de enzimas que hidrolizan los enlaces tipo ester para producir oligómeros solubles en agua, finalmente los microorganismos metabolizan esos productos de degradación en agua y $\mathrm{CO}_{2}$ (Chanprateep, 2010; Valdés et al., 2016). En la región 2850-2960 $\mathrm{cm}^{-1}$ se observaron las bandas correspondientes a las vibraciones de estiramiento de enlaces $\mathrm{CH}, \mathrm{CH}_{2}$ y $\mathrm{CH}_{3}$ del $\mathrm{P}(3 \mathrm{HB}$ ) (Abdelrazek et al., 2016; Nwinyi y Owolabi, 2017), las vibraciones de flexión para estos grupos intervalo $1361-1473 \mathrm{~cm}^{-1}$ no fueron detectadas por el espectrómetro.

\section{Efecto de la biodegradación inicial sobre la fase cristalina}

$\mathrm{P}(3 \mathrm{HB})$ y $\mathrm{PCL}$ son biopolímeros semicristalinos (Abdelrazek et al., 2016; Kovalcik et al., 2017), el primero tiene cerca de $60 \%$ de cristalinidad (lqbal, 2015) y ambos forman arreglos moleculares orto-rómbicos. $\mathrm{P}(3 \mathrm{HB})$

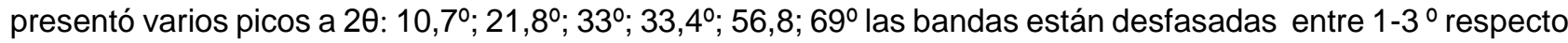
a reportes encontrados en la literatura (lqbal, 2015; Salgaonkar y Bragança, 2017) lo cual es debido a irregularidades morfológicas de la superficie. El difractrograma de PCL (ver Figura 6), tiene tres picos fuertes en 20: 24,8;ํㅜ 25,16우 27,3ํ, lo cual es típico para este biopolímero (Yilgör et al., 2016).

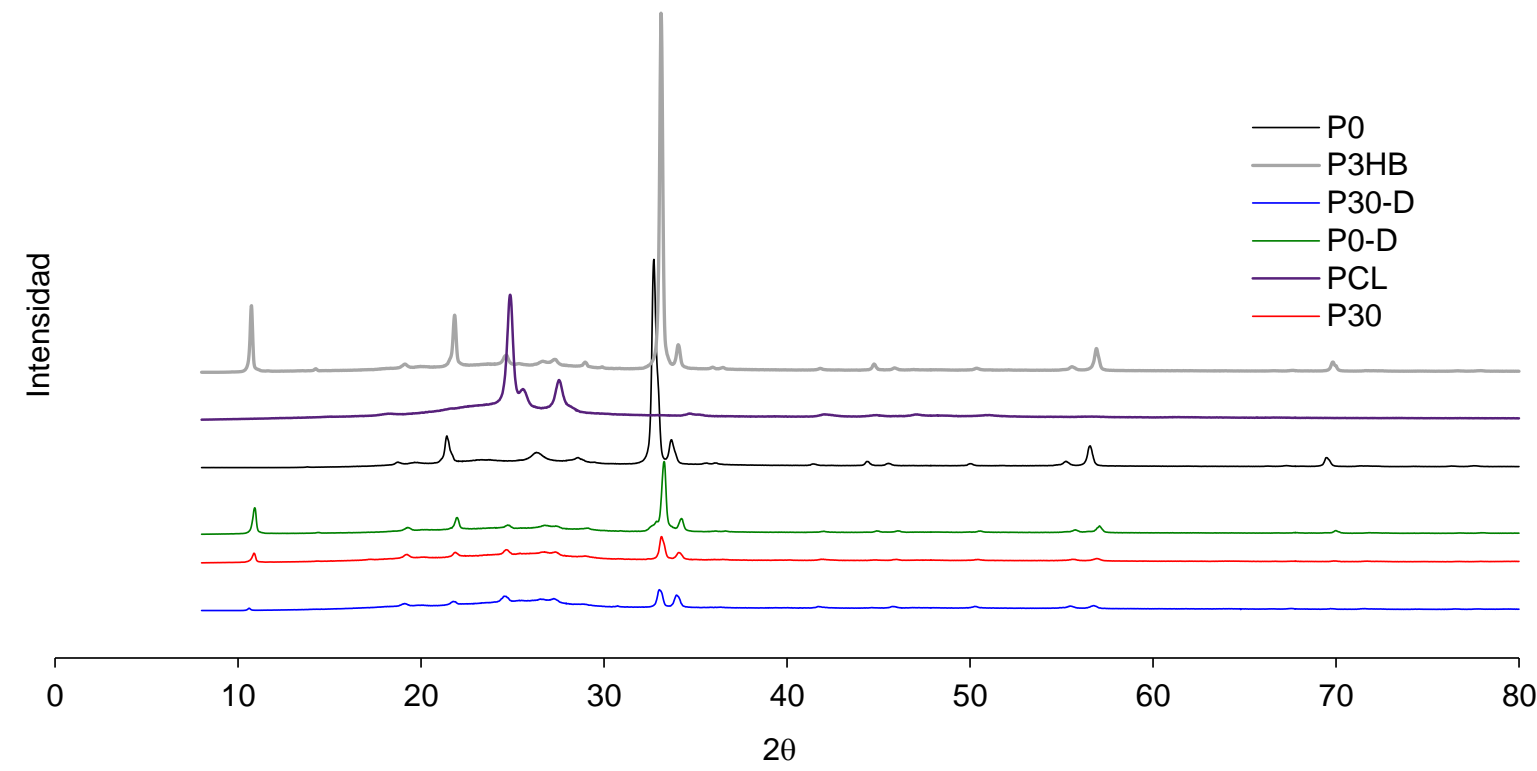

Fig. 6: XDR. Difractograma comparativo de P0, P30 antes y después de biodegradación

El difractograma P0 claramente muestra la contribución de ambos biopolímeros en relación 70:30. P0-D presentó menor intensidad en los picos a $2 \theta: 233$ y 34, es claro que la intensidad de los picos disminuyó, en ese sentido se puede mencionar que hay disminución de la cristalinidad, sin embargo, no se apreció aumento en el ancho de la banda lo cual es característico en polímeros cuando se incrementa la fase amorfa.

En P30, la presencia de ACANB, no permitió distinguir variaciones significativas en los difractogramas antes y después de biodegradación en suelo. Esto ocurrió porque cuando se incorporan materiales hidrófilos como fibras o harinas naturales a matrices hidrófobas se afecta negativamente la estructura cristalina de los polímeros, los grupos hidroxilo perturban las interacciones hidrogeno-hidrogeno y eventualmente conllevan disminución de la cristalinidad de los compuestos (lqbal, 2015, corrobora que los grupos $\mathrm{OH}$ de la celulosa interactúan con PHAs disminuyendo la cristalinidad). Otro estudio señala que al mezclar PCL con almidón también se disminuye la fase cristalina del compuesto (Ali Akbari Ghavimi et al., 2015). 


\section{Análisis morfológico}

La inspección visual de la Figura 7 evidencia cambios morfológicos en las probetas P0-D, P30-D, debidos a biodegradación. Se formaron manchas de color negro, marrón, violeta, poros y rugosidad visibles en las imágenes P0-D y P30-D.

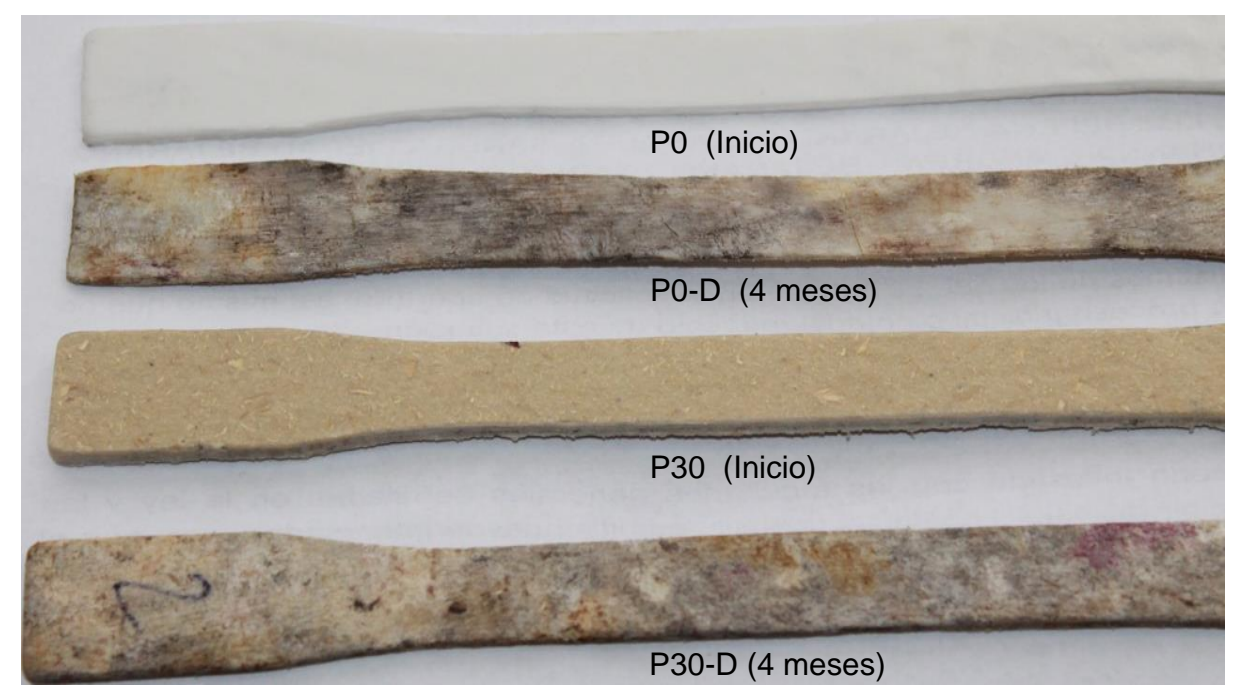

Fig. 7: Imagen de las probetas de tensión, antes y después de biodegradación P0, P30, P0-D, P30-D

Más rigurosamente, con microscopía SEM (ver Figura 8) se observa la erosión superficial con formación de huecos y fisuras. Es allí donde generalmente los microorganismos atacan primero el compuesto, secretando enzimas que depolimerizan los biopolímeros (Boyandin et al., 2013).

Inicio
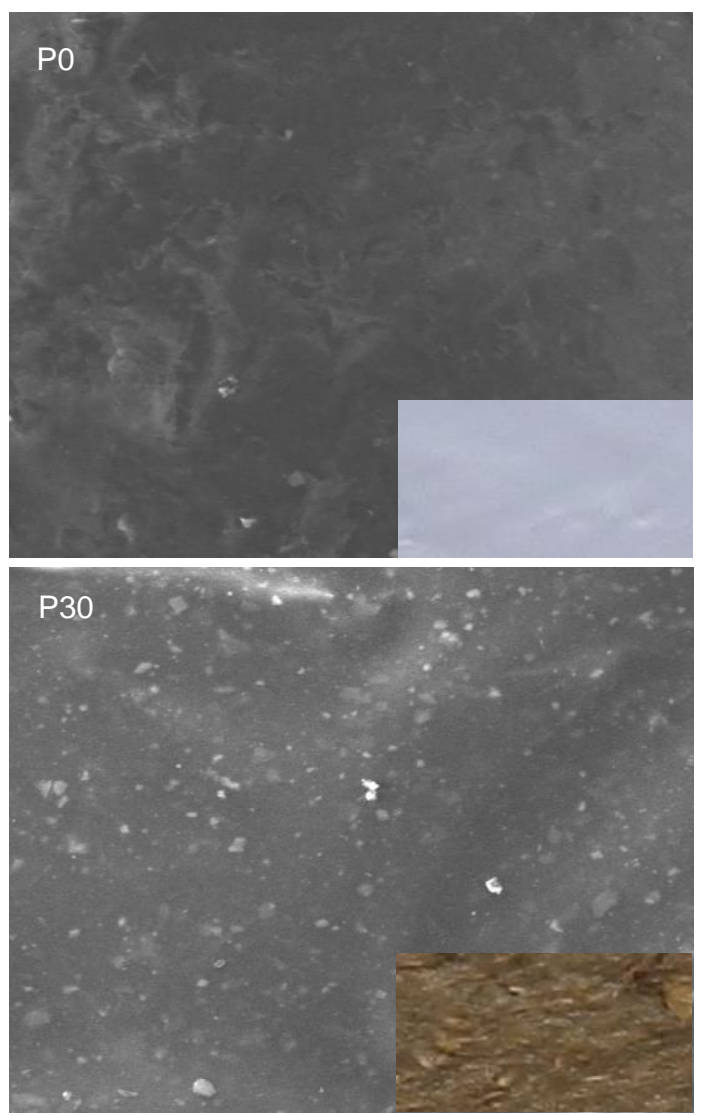

4 Meses
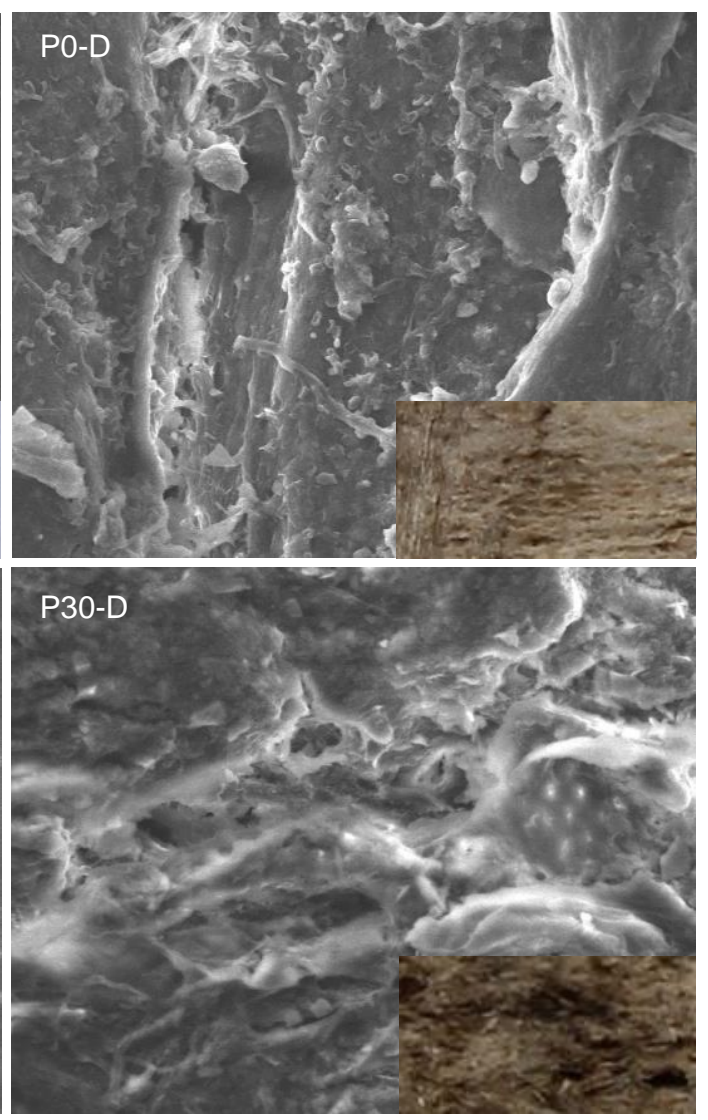

Fig. 8: Imágenes SEM 500x tomadas a la superficie de probetas P0, P30, P0-D, P30-D 


\section{CONCLUSIONES}

Luego de un lapso de cuatro meses, se evidenció biodegradación en biocompuestos elaborados con polihidroxibutirato (70 pcp), policaprolactona (30 pcp) y aserrín de cannabis indica-rusia blanca (0 y 30pcp). Las muestras enterradas en suelo arcilloso sufrieron degradación de su morfología superficial y estructura química (verificada con FT-IR, XDR), disminuyeron sus propiedades de tensión y perdieron masa. La incorporación de 30 partes de aserrín de cannabis indica-rusia blanca afectó negativamente las propiedades de resistencia a la tracción y deformación a rotura, por lo que se requiere la adición de agentes de acoplamiento y tratamientos superficiales para mejorar la compatibilidad entre las partículas de aserrín y los biopolímeros. El módulo de elasticidad se incrementó debido al aporte de rigidez que ofrecen las fibras naturales. Otro hallazgo interesante fue la disminución en $10 \%$ del peso del compuesto al agregar 30 pcp de aserrín respecto al material elaborado únicamente con biopolímeros.

\section{AGRADECIMIENTOS}

Producto derivado del proyecto INV-ING-2385 financiado por la Vicerrectoría de Investigaciones de la Universidad Militar Nueva Granada UMNG-vigencia 2017.

\section{REFERENCIAS}

Abdelrazek, E.M., A. Hezma, A. El-khodary y A. Elzayat, Spectroscopic Studies and Thermal Properties of PCL/PMMA Biopolymer Blend, doi:10.1016/J.EJBAS.2015.06.001, EJBAS 3(1), 10-15 (2016)

Ali, Z., S. Abid e I. Banat, Polyhydroxyalkanoates: Characteristics, Production, Recent Developments and Applications, doi:10.1016/J.IBIOD.2017.10.001, Int. Biodeter. Biodegr., 126, 45-56 (2018)

Ali, S., M. Ebrahimzadeh, M. Solati-Hashjin y N. Abu Osman, Polycaprolactone/Starch Composite: Fabrication, Structure, Properties, and Applications, doi:10.1002/jbm.a.35371, J. Biomed. Mater. Res. A, 103(7), 2482-2498 (2015)

Boyandin, A., S. Prudnikova, V. Karpov y V. Ivonin, Microbial Degradation of Polyhydroxyalkanoates in Tropical Soils, doi:10.1016/J.IBIOD.2013.04.014, Int. Biodeter. Biodegr., 83, 77-84 (2013)

Chanprateep, S., Current Trends in Biodegradable Polyhydroxyalkanoates, doi:10.1016/J.JBIOSC.2010.07.014, J. Biosci. Bioeng., 110(6), 621-632 (2010)

Cirovic, N., M. Kecmanovic, D. Keckarevic y M. Keckarevic, Differentiation of Cannabis Subspecies by THCA Synthase Gene Analysis Using RFLP, doi:10.1016/J.JFLM.2017.07.015, J. Forensic Leg. Med., 51, 81-84 (2017)

Cocca, M., R. Avolio y otros cuatro autores, Amorphized Cellulose as Filler in Biocomposites Based on Poly(Ecaprolactone), doi:10.1016/J.CARBPOL.2014.11.024, Carbohyd. Polym., 118, 170-182 (2015)

Correa, J., V. Molina y otros cuatro autores, Improving Ham Shelf Life with a Polyhydroxybutyrate/Polycaprolactone Biodegradable Film Activated with Nisin, doi:10.1016/J.FPSL.2016.11.004, Food Packaging and Shelf Life, 11, 31-39 (2017)

Coutinho de Paula, F., S. Kakazu y otros tres autores, Polyhydroxyalkanoate Production From Crude Glycerol by Newly Isolated Pandoraea SP, doi:10.1016/J.JKSUS.2016.07.002, J. King Saud University-Science, 29(2), 166-173 (2017)

Iqbal, H., Development of Bio-Composites with Novel Characteristics Through Enzymatic Grafting, Ph.D. Thesis, Faculty of Science and Technology, University of Westminster, London, U.K. (2015)

Ke, Y., X. Zhang y otros tres autores, Reactive Blends Based on Polyhydroxyalkanoates: Preparation and Biomedical Application, doi:10.1016/J.MSEC.2016.03.114, Mater. Sci. Eng. C, 70, 1107-1119 (2017)

Kovalcik, A., K. Meixner, M. Mihalic y W. Zeilinger, Characterization of Polyhydroxyalkanoates Produced by Synechocystis Salina from Digestate Supernatant, doi:10.1016/J.IJBIOMAC.2017.04.054, Int. J. Biol. Macromol., 102, 497-504 (2017)

Kwon, H.J., J. Sunthornvarabhas, J. Park y J. Lee, Tensile Properties of Kenaf Fiber and Corn Husk Flour Reinforced Poly (Lactic Acid) Hybrid Bio-composites: Role of Aspect Ratio of Natural Fibers, doi:10.1016/J.COMPOSITESB.2013.08.003, Composites B, 56, 232-237 (2014)

Liu, M., A. Thygesen, J. Summerscales y A. Meyer, Targeted Pre-Treatment of Hemp Bast Fibres for Optimal Performance in Biocomposite Materials: A Review, doi:10.1016/J.INDCROP.2017.07.027, Ind. Crop. Prod., 108, 660-683 (2017)

López, M.R., J. Alba, J. Gracida y F. Pérez, Production of Polyhydroxyalkanoates (PHAs) with Canola Oil as Carbon Source, doi:10.1016/J.IJBIOMAC.2010.09.016, Int. J. Biol. Macromol., 48(1), 74-80 (2011)

Loureiro, N.C., J. Esteves, J. Viana y S. Ghosh, Development of Polyhydroxyalkanoates/Poly(Lactic Acid) Composites Reinforced with Cellulosic Fibers, doi:10.1016/J.COMPOSITESB.2014.01.001, Compos. Part B: Eng., 60, 603-611 (2014)

Meeks, D., T. Hottle, M. Bilec y A. Landis, Compostable Biopolymer Use in the Real World: Stakeholder Interviews to Better Understand the Motivations and Realities of Use and Disposal in the US, doi:10.1016/J.RESCONREC.2015.10.022, Resour. Conserv.Recy., 105, 134-142 (2015)

Mei, L.H. y N. Oliveira, Caracterização de um Compósito Polimérico Biodegradável Utilizando Poli (E-Caprolactona) e Borra de Café, doi:10.1590/0104-1428.2139, Polímeros, 27(spe), 99-109 (2017) 
Mohanty, A.K., M. Misra y G. Hinrichsen, Biofibres, biodegradable polymers and biocomposites: An overview, doi:10.1002/(SICI)1439-2054(20000301)276:1<1::AID-MAME1>3.0.CO;2-W, Macromol. Mater. Eng., 276-277(1), 1-24 (2000)

Montano-Herrera, L., S. Pratt y M. Arcos-Hernandez, In-Line Monitoring of Thermal Degradation of PHA During MeltProcessing by Near-Infrared Spectroscopy, doi:10.1016/J.NBT.2013.10.005, New Biotechnol., 31(4), 357-363 (2014)

Nwinyi, O. y T. Owolabi, Scanning Electron Microscopy and Fourier Transmission Analysis of Polyhydroxyalkanoates Isolated from Bacteria Species from Abattoir in Ota, Nigeria, doi:10.1016/J.JKSUS.2017.08.003, Journal of King Saud University - Science (2017)

Palacio, O., O. Buitrago y E. Delgado, Evaluación de Polietileno Maleatado en Compuestos de Etil Vinil Acetato y Harina Telinne Monspessulana, doi: 10.4067/S0718-07642016000100015, Inf. Tecnol., 27(1), 139-146 (2016)

Rahman, M., Y. Chen y T. Belsham, Fineness and Tensile Properties of Hemp (Cannabis Sativa L.) Fibres, doi:10.1016/J.BIOSYSTEMSENG.2010.10.004, Biosyst. Eng., 108(1), 9-17 (2011)

Salgaonkar, B.B. y J. Bragança, Utilization of Sugarcane Bagasse by Halogeometricum Borinquense Strain E3 for Biosynthesis of Poly(3-Hydroxybutyrate-co-3-Hydroxyvalerate), doi:10.3390/bioengineering4020050, Bioengineering, 4(2), 50 (2017)

Scaffaro, R., M. Morreale, G. Re y L. Mantia, Degradation of Mater-Bi®/Wood Flour Biocomposites in Active Sewage Sludge, doi:10.1016/J.POLYMDEGRADSTAB.2009.04.028, Polym. Degrad. Stabil., 94(8),1220-1229 (2009)

Singha, A.S. y A. Rana, A Study on Benzoylation and Graft Copolymerization of Lignocellulosic Cannabis Indica Fiber, doi:10.1007/s10924-011-0370-9, J. Polym. Environ., 20(2), 361-371 (2012)

Srinivasababu, N., Assessing the Mechanical Performance Cannabis Sativa Composites - Reinforced with Long Time Dried Fibre, doi:10.1016/J.PROENG.2014.12.375, Procedia Engineering, 97, 986-993 (2014)

Sun, S., P. Liu y otros tres autores, Effects of Low Polyhydroxyalkanoate Content on the Properties of Films Based on Modified Starch Acquired by Extrusion Blowing, doi:10.1016/J.FOODHYD.2017.05.030, Food Hydrocolloids, 72, 81-89 (2017)

Urtuvia, V., P. Villegas, M. González y M. Seeger, Bacterial Production of the Biodegradable Plastics Polyhydroxyalkanoates, doi:10.1016/J.IJBIOMAC.2014.06.001, Int. J. Biol. Macromol. 70, 208-213 (2014)

Valdés, A., O. Fenollar y A. Beltran, Characterization and Enzymatic Degradation Study of Poly( $(\varepsilon$-Caprolactone)-Based Biocomposites from Almond Agricultural by-Products, doi:10.1016/J.POLYMDEGRADSTAB.2016.02.023, Polym. Degrad. Stabil., 132, 181-190 (2016)

Vanegas, D. y M. Ramírez, Correlación del Crecimiento de Pseudomonas Fluorescens en la Producción de Polihidroxialcanoatos de Cadena Media (PHAMCL) Mediante Modelos Primarios de Gompertz, Logístico y Baranyi, doi: 10.4067/S0718-07642016000200011, Inf. Tecnol., 27(2), 87-96 (2016)

Volova, T.G., A. Boyandin y otros tres autores, Biodegradation of Polyhydroxyalkanoates (PHAs) in Tropical Coastal Waters and Identification of PHA-Degrading Bacteria, doi:10.1016/J.POLYMDEGRADSTAB.2010.08.023, Polym. Degrad. Stabil., 95(12), 2350-2359 (2010)

Wachirahuttapong, S., C. Thongpin y N. Sombatsompop, Effect of PCL and Compatibility Contents on the Morphology, Crystallization and Mechanical Properties of PLA/PCL Blends, doi:10.1016/J.EGYPRO.2016.05.026, Energy Procedia, 89, 198-206 (2016)

Yean, S. y K. Sudesh, Effects of Polyhydroxyalkanoate Degradation on Soil Microbial Community, doi:10.1016/J.POLYMDEGRADSTAB.2016.06.024 Polym. Degrad. Stabil., 131, 9-19 (2016)

Yilgör, E., M. Isik, C. Kosak y L. Yilgör, Synthesis and Structure-Property Behavior of PolycaprolactonePolydimethylsiloxane-Polycaprolactone Triblock Copolymers, doi:10.1016/J.POLYMER.2015.12.024, Polymer, 83, 138153 (2016) 